\title{
TRI-CRO: aplicación para la comprobación, resolución de triángulos rectángulos, oblicuángulos y almacenamiento temporal de datos
}

\section{TRI-CRO: application for the verification, resolution of rectangle triangles, oblicuangles and temporary storage of data}

\author{
Luciano Guevara ${ }^{I^{*}}$, Heidy Calderón ${ }^{1}$, Luis Sánchez ${ }^{1}$, Tejedor Morales María Yahaira ${ }^{l}$ \\ ${ }^{1}$ Universidad Tecnológica de Panamá, Centro Regional de Coclé, Grupo de Investigación SoftSolution Group
}

\begin{abstract}
Resumen Debido a la falta de interés por las matemáticas de los estudiantes en nuestro país se hizo necesario un estudio acerca de las causas de esta problemática, y desarrollar formas de motivar a los estudiantes a mejorar su rendimiento académico. En este artículo se presenta el diseño, desarrollo e implementación de TRI-CRO, una aplicación desarrollada en Java capaz de comprobar, resolver y almacenar problemas matemáticos como: triángulos rectángulos y triángulos oblicuángulos mediante un arreglo de objetos. En primer lugar, se presentan los recursos utilizados en el desarrollo de la aplicación, como lo son el software de desarrollo, algoritmos, fórmulas, las cuales se mencionan: ley del seno, ley del coseno, funciones trigonométricas y teorema de Pitágoras. Seguido la metodología, analiza en primera instancia, captar datos por parte del estudiante, como responde la aplicación, si es efectiva en los resultados que arroja, desarrollada con una interfaz gráfica interactiva con el usuario. Finalmente, se desarrolló una investigación para evaluar la aplicación. Para ello se sometió a prueba con una muestra de 20 estudiantes de primer año de la Universidad Tecnológica de Panamá, Centro Regional de Coclé con la finalidad de medir la efectividad de la aplicación dentro del contenido de la asignatura Matemáticas I, además de promover el aprendizaje de las matemáticas a este nivel educativo, planteando la posibilidad de la implementación de herramientas tecnológicas como estrategia de estudio de los docentes.
\end{abstract}

Palabras clave Triángulos rectángulos, triángulos oblicuángulos, arreglo de objetos, almacenamiento temporal.

\begin{abstract}
Due to the lack of interest in the mathematics of students in our country, it became necessary to study the causes of this problem and develop ways to motivate students to improve their academic performance. This paper presents the design, development and implementation of TRI-CRO, an application developed in Java capable of checking, solving and storing mathematical problems such as: right triangles and oblique triangles through an array of objects. In the first place, the resources used in the development of the application are presented, such as the development software, algorithms, formulas, which are mentioned: sine law, cosine law, trigonometric functions and Pythagoras theorem. Following the methodology, analyze in the first instance, capture data by the student, how the application responds, if it is effective in the results it produces, developed with an interactive graphic interface with the user. Finally, a research was developed to evaluate the application. To this end, a sample of 20 first-year students from the Technological University of Panama, Coclé Regional Center, was tested with the aim of measuring the effectiveness of the application within the contents of the subject Mathematics I, in addition to promoting learning of mathematics at this educational level, raising the possibility of the implementation of techno-logical tools as a strategy for the study of teachers.
\end{abstract}

Keywords Rectangular triangles, oblique triangles, array of objects, temporary storage.

*Corresponding author: luciano.guevara@utp.ac.pa

\section{Introducción}

"Las matemáticas, además de desarrollar la intuición y el espíritu crítico, constituyen un elemento insustituible de formación en el rigor, formalismo y razonamiento" [1].

Se puede evidenciar la importancia que tiene el aprendizaje de las matemáticas en el proceso de formación de los estudiantes. Sin embargo, muchos jóvenes en nuestro país no sienten interés en ellas, convirtiéndose en un gran problema en la actualidad.

En el 2009, la Organización para la Cooperación y Desarrollo Económicos (OCDE), realizó el informe PISA. Este informe se encarga de evaluar la calidad de los estudiantes en cuanto a las matemáticas, ciencias y habilidad lectora, 
quedando nuestro país entre los tres últimos lugares de 65 países que participaron.

El informe PISA es el Programme for International Student Assessment, conocido en español como Programa para la Evaluación Internacional de Estudiantes [2].

Según Guillermo Dávila Rascón "El aprendizaje de las matemáticas se dificulta a niños y jóvenes, pues el modelo de enseñanza utilizado en las aulas es inadecuado para la manera que funciona el cerebro, que no está hecho para memorizar" [3].

Actualmente, los estudiantes no desarrollan sus tareas, por el hecho de no entender la metodología de estudio de sus profesores, estos se retiran a sus hogares con dudas por temor a preguntar sobre ellas, y como pueden tratan de hacer sus prácticas sin tener un medio para comprobar sus respuestas. Si bien existen calculadoras capaces de realizar grandes cálculos, a nivel educativo, carecen de ellas. Por esta razón, el estudio consiste en el desarrollo de Triangles Checker of Rectangular and Oblique TRI-CRO, una aplicación capaz de realizar cálculos matemáticos complejos como lo son: triángulos rectángulos y triángulos oblicuángulos, temas que fueron escogidos dentro del contenido de la asignatura Matemáticas I de la Universidad Tecnológica de Panamá por su nivel de complejidad, en busca de impulsar el aprendizaje de las matemáticas mediante herramientas tecnológicas e implementarlas en las estrategias didácticas de los docentes.

La aplicación, además de realizar dichos cálculos, brinda al usuario la posibilidad de almacenar los datos de cada problema ingresado; asignando automáticamente un código de identificación a cada uno, pudiendo acceder a ellos en cualquier momento durante la ejecución de la aplicación.

Las secciones de la investigación realizada se describen a continuación, iniciando con los antecedentes, luego se presentan los materiales y metodología, seguidamente los resultados obtenidos, para terminar, se presentan las conclusiones y las recomendaciones.

\section{Antecedentes}

Para la elaboración de TRI-CRO, se investigó de antemano las características que presentan los triángulos, por ejemplo:

- Un lado de un triángulo es siempre menor a la suma de los otros dos lados $(a<b+c)$, pero mayor que su diferencia $(\mathrm{a}>\mathrm{b}-\mathrm{c})$.

- La suma de todos los ángulos interiores da siempre $180^{\circ}(\mathrm{A}$ $+\mathrm{B}+\mathrm{C}=180^{\circ}$ ) [4].

De no cumplirse con estas condiciones, no sería llamado triángulo.

Un triángulo rectángulo es aquel que tiene un ángulo recto igual a $90^{\circ}$ y dos ángulos agudos menores de $90^{\circ}$ [5].

Un triángulo es oblicuángulo si ninguno de sus ángulos internos es un ángulo recto, es decir, es aquel que no es rectángulo [6].

Existen algunas aplicaciones que desempeñan este tipo de cálculos, sin embargo, sus funciones carecen de interacción con el usuario [7]. Esto abrió la posibilidad de mejorar e implementar el nuevo algoritmo para realizar cálculos de manera más dinámica y eficiente, utilizando una estructura de datos como lo es un arreglo de objetos utilizado para el almacenamiento de los resultados obtenidos en cada problema, mediante el lenguaje de programación Java.

\section{Materiales y métodos}

En esta sección se muestran los materiales y la metodología utilizada para el desarrollo de TRI-CRO.

\subsection{Software}

Durante la programación de la aplicación se utilizó el entorno integrado de desarrollo NetBeans en su versión más reciente 8.2 de la compañía Oracle, la cual permite el uso de diferentes lenguajes de programación, siendo Java el escogido por la particularidad de ser orientado a objetos.

"La tecnología orientada a objetos se define como una metodología de diseño de software que modela las características de objetos reales o abstractos por medio del uso de clases y objetos" [8].

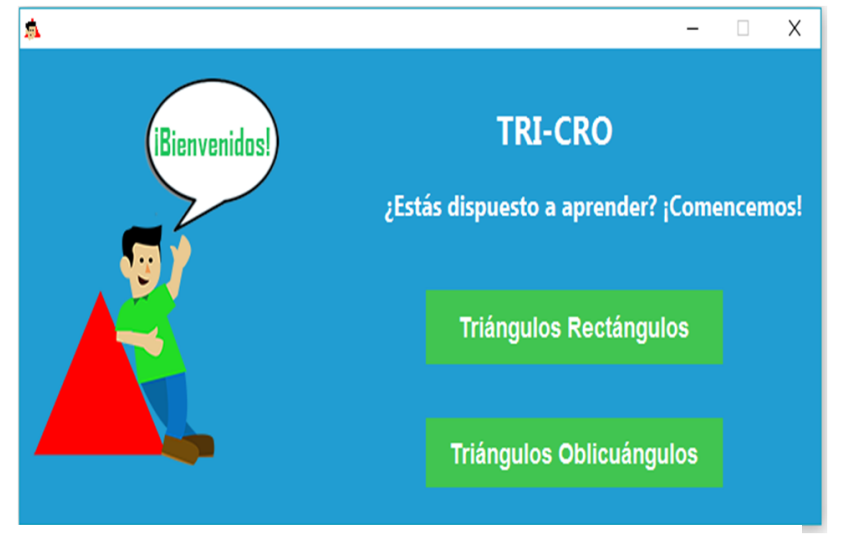

Figura 1. Menú principal de TRI-CRO.

\subsection{Obtención de datos}

Los ángulos y lados de cada triángulo son ingresados por el usuario, los cuales son obtenidos por medio de la interfaz gráfica de Java diseñada con formularios JFrame, esta ventana brinda al usuario la posibilidad de escoger el caso del problema, habilitando los campos para el ingreso de este, posteriormente distribuidos a las clases donde se realizan los cálculos. 


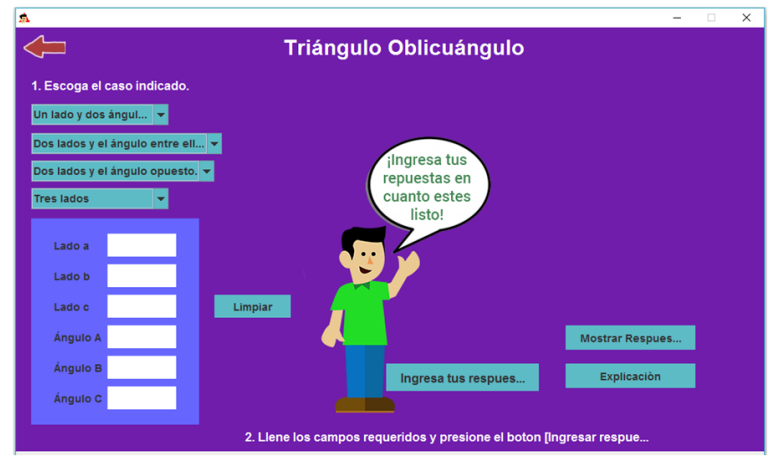

Figura 2. Interfaz gráfica para el ingreso y comprobación de triángulos oblicuángulos.

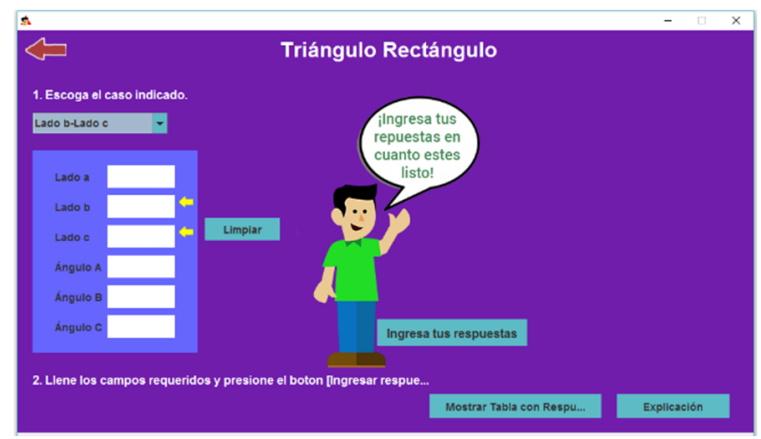

Figura 3. Interfaz para el ingreso de datos y comprobación de triángulos rectángulos.

\subsection{Fórmulas}

Para hacer los cálculos de las diferentes operaciones que posee TRI-CRO, se tradujeron las fórmulas del lenguaje matemático convencional a un lenguaje de programación, haciendo uso de la librería Math que tiene Java. Como lo muestran los siguientes recuadros:

Public double AngCos(double lado 1, double lado2, double lado3)\{

Double ang $=$ (Math.pow (lado1,2) + Math.pow(lado2,2)-

Math.pow(lado3,3))/(2*lado1*lado2);

Doublé angulo= Math.acos(ang)/Math.PI/180;

return angulo;

Public double AngSen (double lado1, double lado2, double angO) \{

Double ang $=(($ Math.sin $(\operatorname{ang} O *$ Math.PI/180 $) *$ ladol $))$ (lado2);

Double angulo $1=$ Math. asin(ang)/Math.PI/180;

Return angulo1;

Figura 4. Fórmulas matemáticas traducidas a lenguaje de programación Java.
Public double LadoSen(double ladol, double angl, double ang2) \{

double lado=(Math.sin(ang2*Math.PI/180)*lado1)

(Math.sin(angl *Math.PI/180));

Return lado;\}

Public double LadoCos (double lado1, double lado 2,

double angO)\{

Double lado $=$

Math.sqrt(Math.pow(lado1,2)+Math.pow(lado2,2)-

$2 *$ ladol *lado $2 *$ Math.cos(angO*Math.PI/180)); Ret

urn lado; $\}$

Figura 5. Otras fórmulas matemáticas traducidas a lenguaje de programación Java.

\subsubsection{Teorema de Pitágoras}

Fórmula general del teorema de Pitágoras para el cálculo de la hipotenusa si es ingresado los catetos a y b en triángulos rectángulos.

$$
c^{2}=a^{2}+b^{2}
$$

\subsubsection{Funciones Trigonométricas}

Fórmulas para el cálculo de triángulos rectángulos si es ingresado un lado y un ángulo.

$$
\begin{aligned}
& \operatorname{sen} \emptyset=\frac{a}{c} \sec \emptyset=\frac{b}{c} \tan \emptyset=\frac{a}{b} \\
& \csc \emptyset=\frac{c}{a} \sec \emptyset=\frac{c}{b} \cot \emptyset=\frac{b}{a}
\end{aligned}
$$

\subsubsection{Ley del Seno}

Fórmula utilizada para evaluar triángulos oblicuángulos cuando se dan los casos siguientes:

1. Dado un lado y dos ángulos.

2. Dado dos lados y el ángulo opuesto a uno de ellos.

$$
\frac{a}{\operatorname{sen} A}=\frac{b}{\operatorname{sen} B}=\frac{c}{\operatorname{sen} C}
$$

\subsubsection{Ley del Coseno}

Fórmula empleada para los siguientes casos de triángulos oblicuos:

1. Dado dos lados y el ángulo entre ellos.

2. Dado tres lados y ningún ángulo.

$$
a=\sqrt{b^{2}+c^{2}-2 b c \cos A}
$$




$$
\begin{aligned}
& b=\sqrt{a^{2}+c^{2}-2 a c \cos B} \\
& c=\sqrt{a^{2}+b^{2}-2 a b \cos C}
\end{aligned}
$$

\subsection{Cálculos}

Los cálculos se realizan dentro de dos clases distintas, primeramente, la clase TrianguloRect para los triángulos rectángulos y segundo la clase TrianguloOblic para los oblicuángulos. Cada una posee atributos y métodos donde se envían los valores ingresados por el usuario desde la interfaz gráfica (métodos set()) encargados de asignar los datos en donde se efectúan los cálculos internamente sin mostrarlos, para luego comprobar las respuestas obtenidas por el usuario, de no coincidir, muestra en pantalla mediante (métodos get()) los resultados de cada lado y ángulo del problema presentado:

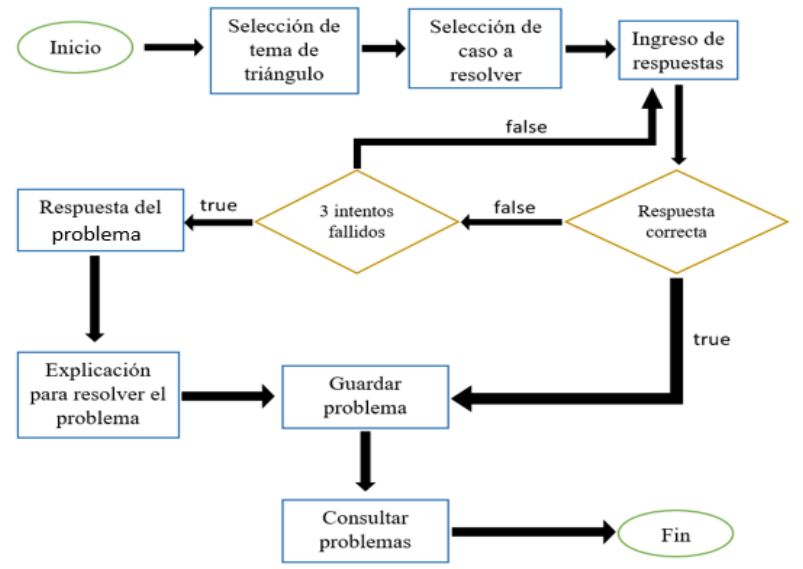

Figura 6. Diagrama de flujo de TRI-CRO.

\subsection{Funcionalidad}

TRI-CRO permite escoger qué tipo de triángulo va a introducir. Dentro de las funcionalidades de los triángulos rectángulos y oblicuángulos, se despliegan todos los casos posibles, de manera que le sea sencillo elegir al usuario, habilitando solo los campos donde debe ingresar los datos, calculando los lados, ángulos y funciones trigonométricas de cada triángulo sin mostrarlos, luego el usuario debe ingresar sus respuestas, las cuales son comparadas con los datos ingresados en principio, mandando un mensaje indicando si está correcta o no la respuesta. De no estar bien la respuesta, se despliega un mensaje incitando al usuario a intentarlo nuevamente, pasado el tercer intento se habilita el botón de respuesta, el cual mostrará los resultados de cada problema.

Finalmente, permite al usuario almacenar mediante un arreglo de objetos los resultados de cada operación de forma temporal durante el tiempo de ejecución del programa, asignando automáticamente a cada problema un código de identificación y así obtener los resultados previos sin tener que volver a ingresar los datos, siendo esta última de gran ayuda en cuanto al ahorro de tiempo en la realización de las tareas.

\subsection{Implementación}

TRI-CRO fue sometida a prueba analizando problemas de triángulos rectángulos y oblicuángulos, midiendo así la factibilidad en los resultados, de manera que el usuario al ingresar sus datos compruebe las respuestas con total confiabilidad.

Luego, se realizó dos encuestas con una muestra de 20 estudiantes de primer año de la Universidad Tecnológica de Panamá C.R. de Coclé dentro de la asignatura Matemáticas I, para obtener información acerca de la razón principal por la cual se les dificulta el aprendizaje de las matemáticas, también, si una herramienta tecnológica podría motivar y ayudar a comprender mejor esta materia, por último, se midió la efectividad de la aplicación, permitiendo a los estudiantes el uso de ella.

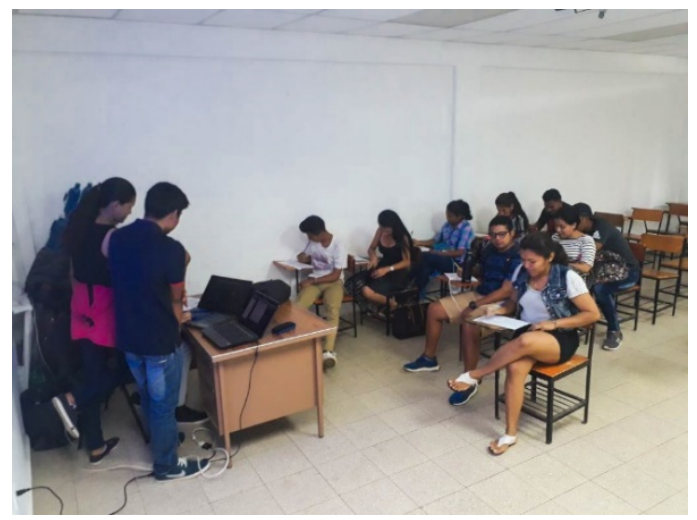

Figura 7. Estudiantes encuestados.

\section{Resultados}

En las pruebas realizadas a TRI-CRO, se comprobaron respuestas a problemas de todos los casos posibles de triángulos rectángulos y oblicuángulos, acertando en cada uno de ellos.

Los resultados de las encuestas realizadas a los 20 estudiantes de primer año se muestran en la tabla 1 y 2 .

Tabla 1. Resultados relevantes de la encuesta

\begin{tabular}{|l|c|c|c|c|}
\hline \multicolumn{5}{|c|}{ Resultados de la encuesta } \\
\hline $\begin{array}{l}\text { ¿Por qué se les } \\
\text { dificulta el } \\
\text { aprendizaje y } \\
\text { entendimiento de } \\
\text { las matemáticas? }\end{array}$ & $\begin{array}{l}\text { Temor por } \\
\text { preguntar }\end{array}$ & $\begin{array}{c}\text { Metodología del } \\
\text { profesor }\end{array}$ & $\begin{array}{c}\text { Falta de } \\
\text { confianza } \\
\text { con el } \\
\text { docente }\end{array}$ & $\begin{array}{c}\text { Falta de } \\
\text { motivación }\end{array}$ \\
\cline { 2 - 5 } & $4(20 \%)$ & $10(15 \%)$ & $3(15 \%)$ & $3(15 \%)$ \\
\hline \begin{tabular}{l} 
¿Una $\begin{array}{l}\text { Serramimenta } \\
\text { tecnológica les } \\
\text { ayudaría a } \\
\text { comprender mejor } \\
\text { las matemáticas? }\end{array}$ \\
\cline { 2 - 5 }
\end{tabular} & \multicolumn{2}{|c|}{ No } \\
\hline
\end{tabular}


Se obtuvo que la dificultad más común que presentan los estudiantes con las matemáticas se debe a la metodología de estudio del docente, de igual forma, se alcanzó una favorable aceptación del uso de este tipo de herramientas tecnológicas para ayudar a motivar y comprender esta materia.

Tabla 2. Resultados de la encuesta

\begin{tabular}{|l|c|c|}
\hline \multicolumn{3}{|c|}{ Resultados de la encuesta } \\
\hline & $\begin{array}{c}\text { Fácil de usar, diseño } \\
\text { ¿Cómo les pareció } \\
\text { la aplicación? }\end{array}$ & $\begin{array}{c}\text { No fue tan sencillo de } \\
\text { utilizar }\end{array}$ \\
\cline { 2 - 3 } & $15(75 \%)$ & $5(25 \%)$ \\
\hline
\end{tabular}

A los estudiantes que hicieron uso de TRI-CRO, primeramente, se les explicó brevemente su funcionamiento, luego, sabiendo manejar solos la aplicación, comprobaron algunos problemas.

De los encuestados, un $75 \%$ afirmó que la misma posee un diseño agradable y fácil de entender.

Mientras que el 25\% dijo que no fue tan sencilla de utilizar, recibiendo sugerencias tales como:

- Cambiar el tipo de fuente de las instrucciones.

- Utilizar colores más llamativos.

Sin embargo, la aplicación fue recibida de una manera muy positiva.

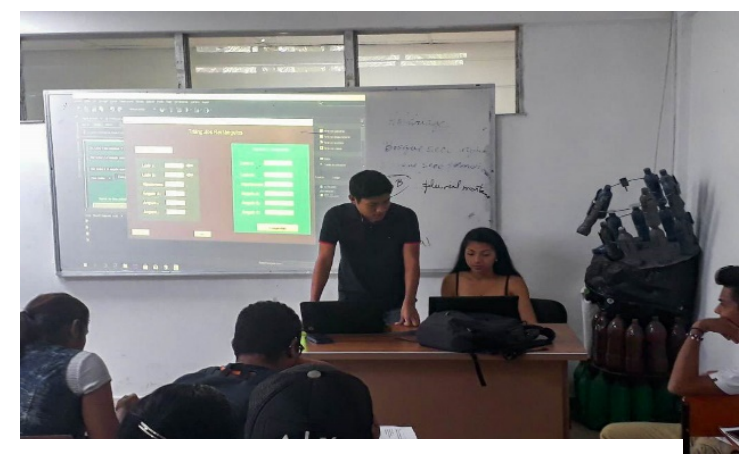

Figura 8. Estudiantes probando la aplicación.

Por último, se sugirió la posibilidad del uso de TRI-CRO dentro de las estrategias de estudio de la profesora de Matemáticas I de la Universidad Tecnológica de Panamá Centro Regional de Coclé, Julia Lombardo, a la hora de dar inicio con los temas abordados por la aplicación. Siendo aceptada la propuesta se realizaron prácticas y talleres utilizando la aplicación como parte de las asignaciones dadas por la profesora a los estudiantes.

\section{Conclusiones}

Se logró implementar a TRI-CRO como un recurso tecnológico de la profesora de matemáticas de la Universidad Tecnológica de Panamá C.R. de Coclé Julia Lombardo, dentro de la asignatura Matemáticas I, impulsando el aprendizaje de las matemáticas, motivando a los estudiantes a desarrollar sus prácticas, teniendo un modo de comprobar sus respuestas.

El diseño de la interfaz fue percibido como agradable y fácil de utilizar por los estudiantes que formaron parte de las pruebas de la aplicación.

Las encuestas realizadas, pueden ser utilizadas para futuras referencias en proyectos e investigaciones vinculadas con herramientas tecnológicas asociadas al estudio de las matemáticas a este nivel educativo.

\section{Recomendaciones}

- Desarrollar nuevas metodologías y estrategias de estudio más dinámicas, utilizando herramientas tecnológicas.

- Mejorar el diseño de la aplicación, habilitando otras funciones para la comprobación de respuestas.

- Desarrollar métodos para mostrar gráficamente cada triángulo en forma precisa.

- Utilizar otro tipo de estructuras de datos para almacenar de manera distinta los resultados de las operaciones.

- Implementar la aplicación también para dispositivos móviles con sistema operativo Android.

\section{AGRADECIMIENTO}

A la profesora Julia Lombardo de la Universidad Tecnológica de Panamá, Centro Regional de Coclé, por el espacio y tiempo brindado con sus estudiantes al momento de la recopilación de datos y pruebas.

\section{REFERENCIAS}

[1] J. Sepulcre. (2013, Jun.). "Matemáticas en la vida cotidiana", [Online].

Available: http://www.diarioinformacion.com/opinion/2013/06/15/matem aticas-vida-cotidiana/1385553.html [Accessed: Mayo: 2018].

[2] Organización para la Cooperación y Desarrollo Económicos. (2018). "PISA", [Online]. Available: https://www.oecd.org/pisa/aboutpisa/ [Accessed: Mayo: 2018].

[3] G. Dávila. (2013, Aug.). "Aprendizaje de matemáticas se dificulta por enseñanza inadecuada en aulas", [Online]. Available: http://pulsoslp.com.mx/2013/08/07/aprendizaje-dematematicas-se-dificulta-por-ensenanza-inadecuada-en-aulasexperto/ [Accessed: Mayo: 2018].

[4] S. Lasso. (2016, May.). "Triángulos, que es, características y fórmulas", [Online]. Available: https://www.aboutespanol.com/triangulo-que-es-

caracteristicas-y-formulas-180131 [Accessed: Mayo: 2018].

[5] Centro de Estudios, Didáctica y Capacitación. Academia y tecnología. (2015). "Resolución de triángulos rectángulos y oblicuángulos", [Online]. pp. 2. Available: http://cedicap.ailosabe.com/PDF/Resoluci\%C3\%B3n $\% 20 \mathrm{de} \%$ 20Tri\%C3\%A1ngulos.-\%20CEDICAPED.pdf [Accessed: Mayo: 2018].

[6] Centro de Estudios, Didáctica y Capacitación. Academia y tecnología. (2015). "Resolución de triángulos rectángulos y oblicuángulos", [Online]. pp. 4. Available: 
Guevara (et al): TRI-CRO: Aplicación para la comprobación, resolución de triángulos rectángulos, oblicuángulos y almacenamiento temporal de datos

http://cedicap.ailosabe.com/PDF/Resoluci $\% \mathrm{C} 3 \% \mathrm{~B} 3 \mathrm{n} \% 20 \mathrm{de} \%$ 20Tri\%C3\%A1ngulos.-\%20CEDICAPED.pdf [Accessed: Mayo: 2018].

[7] (2018, Mar.). "Aplicaciones para resolver triángulos rectángulos y oblicuángulos", [Online]. Available: https://www.androidesapk.com/aplicaciones-resolver-

triangulos-rectangulos-oblicuangulos/ [Accessed: Mayo: 2018].

[8] J. De los Ángeles. (2003). "Intro_poo", [Online]. pp. 1. Available:

http://pdi.topografia.upm.es/m.manso/docencia/Informatica_pl an92/Curso-2002-2003/poo.pdf [Accessed: Mayo: 2018]. 